\title{
Sculpt with Optra Pad : A case report
}

\author{
Niladri Maiti ${ }^{1}$, Utpal Kumar Das ${ }^{2}$ \\ ${ }^{1}$ Post Graduate Student, Department of Conservative Dentistry \& Endodontics, Guru Nanak Institute of Dental Science and \\ Research, Kolkata India \\ ${ }^{2} \mathrm{HOD}$, Department of Conservative Dentistry \& Endodontics, Guru Nanak Institute of Dental Science and Research, Kolkata India \\ *Corresponding author E-mail: dr.niladrimaiti@gmail.com
}

\begin{abstract}
Because of the socioeconomic status of the patients, direct resin composite laminate veneers were used to improve the esthetics of their maxillary and mandibular anterior teeth. Laminate veneers are restorations which are envisioned to correct existing abnormalities, esthetic deficiencies and discolorations This case report reflects the use of a innovative composite sculpting instrument ( OPTRA SCULPT PAD) for direct composite laminate in a patient who came with complaint of broken old laminate.
\end{abstract}

Keywords: Optra Sculpt Pad, Direct Composite Veneer.

\section{Introduction}

Patients' demand for esthetic restorations and their increasing desire to preserve remaining sound tooth structure are pushing dentists to broaden the clinical indications for direct resin based composite restorations.[1]The development of adhesive bonding and resin composite systems has increased the popularity of resin composite restorations. The improvement in modern adhesive techniques, enhanced handling characteristics of resin-based composites, and preparation designs allow clinicians to use a more conservative treatment protocol involving a reliable and direct chairside protocol for the restoration of the teeth.[2-4]. Moreover, direct resin composite restorations do not require complex clinical and laboratory procedures. The results achieved can be satisfactory, both esthetically and functionally. Direct composite laminates have better polishability. Such veneers can provide extremely good esthetic results, yet are conservative in nature and a sound alternative to more extensive restorative procedures.[5] A conservative veneer technique involves the application of the resin-based composites without tooth reduction. Furthermore, resin-based composite veneers can be altered and repolished in situ, which is very useful when subtle changes in contour, such as alteration of the emergence profile, are

desired. Also, composite resin veneers are not as expensive when compared to porcelain laminate veneers.[6]

OPTRA SCULPT PAD by IVOCLAR VIVADENT is a modelling instrument designed for the intraoral adaptation, shaping and contouring of uncured packable composite filling materials. OptraSculpt Pad consists of an autoclavable handheld instrument and single use disc-shaped attachments (pads) in diameters ranging from 4 to $6 \mathrm{~mm}$. The handheld instrument contains a metal spatula on one working end and a holding ring on the other.

\section{Case report}

A 35-year-old female patient was referred to the Department of Conservative Dentistry and Endodontics for evaluation and treatment of non-carious lesion involving her anterior teeth(Fig.no.1,2). Prior to treatment, detailed dental, medical, and social histories were obtained. The patient's periodontal status was assessed and found to be otherwise healthy and noncontributory. A treatment plan was developed with the goal of restoring the maxilary left and right central incisors and improving the patient's appearance with direct resin composite laminate veneers. The treatment plan was approved by the patient.

Shade selection was performed .For maxillary anterior teeth, $0.5 \mathrm{~mm}$ of facial reduction was achieved using highspeed water-cooled diamond burs for direct resinbased composite laminate veneers. All tooth preparations were free of sharp internal line angles.(Fig.no.3) 
The teeth was treated with self-etching adhesive system(GLUMA SELF-ETCH,HERAEUS,GERMANY) (Fig.no.4) Then composite resin(TETRIC N CERAM) was used in the restoration of the patient's maxillary anterior teeth with the OPTRA SCULPT PAD (IVOCLAR VIVADENT )(Fig.no.5,6). The composite resin was polymerized using BLUE PHASE for 30 seconds and any residual excess at the restorative margin was removed with a series of finishing burs, followed by polishing to a high lustre using ASTROPOL AND ASTROBRUSH (IVOCLAR VIVADENT) .(Fig.no.7). The patient was recalled approximately after six months for follow-up examinations, no chipping, discoloration, or secondary caries were noted.(Fig.no.8).

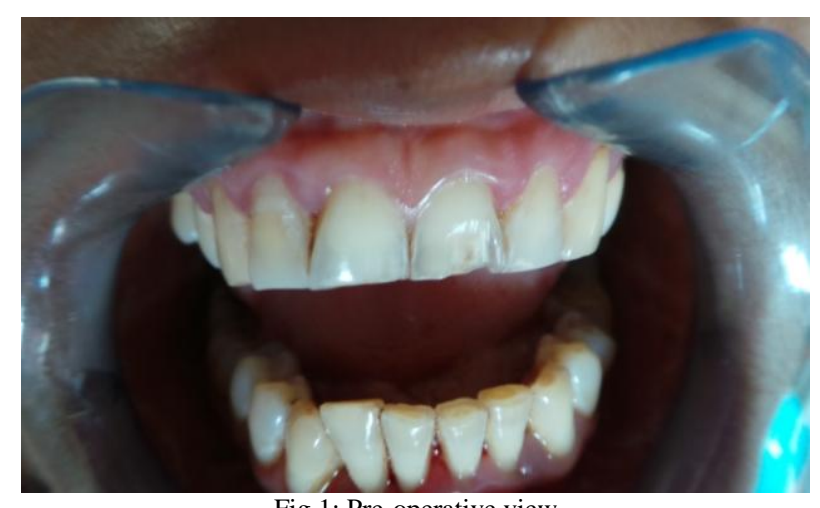

Fig.1: Pre-operative view

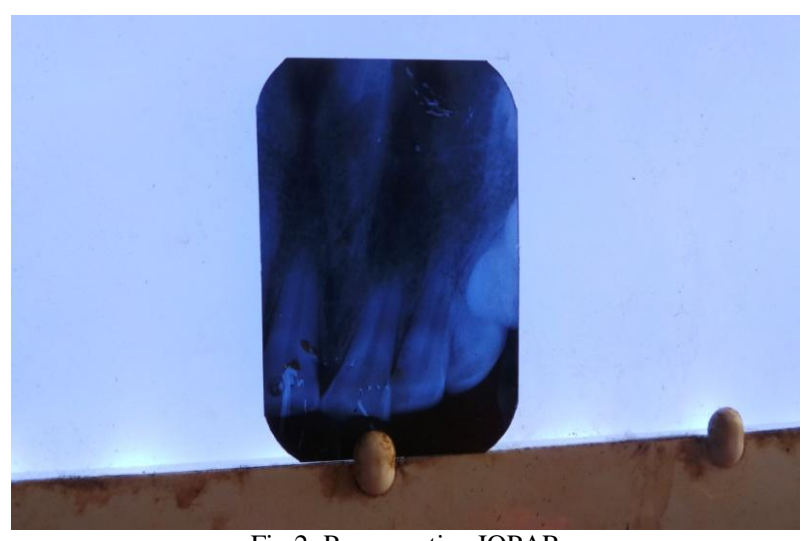

Fig.2: Pre operative IOPAR

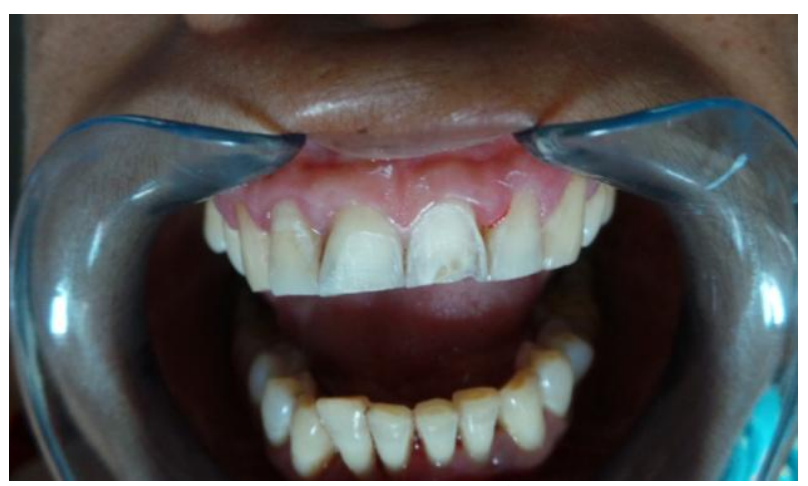

Fig.3: Tooth preparation 


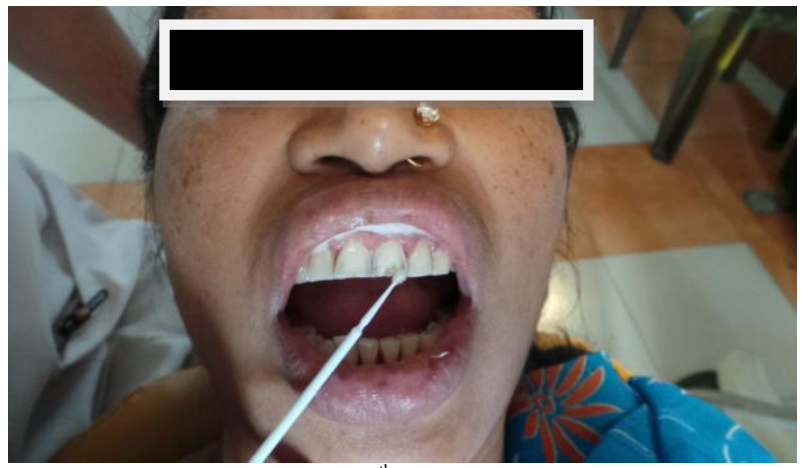

Fig.4: Application of $7^{\text {th }}$ generation bonding agent
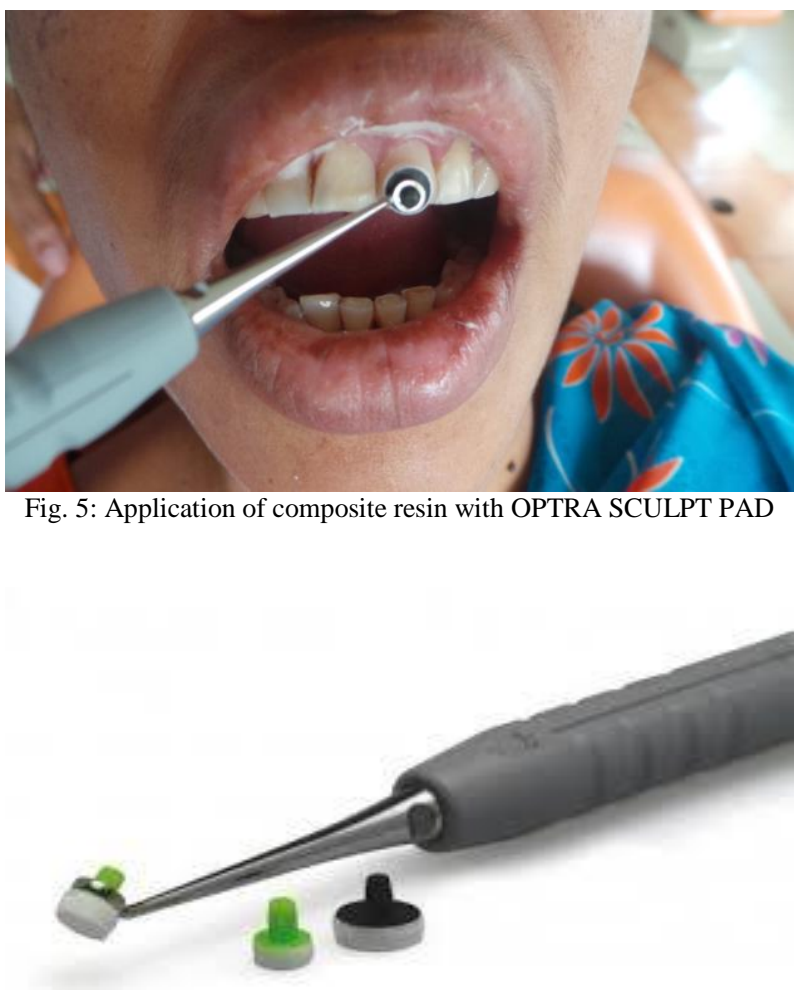

Fig.6: Optra Sculpt Pad with disposable tips

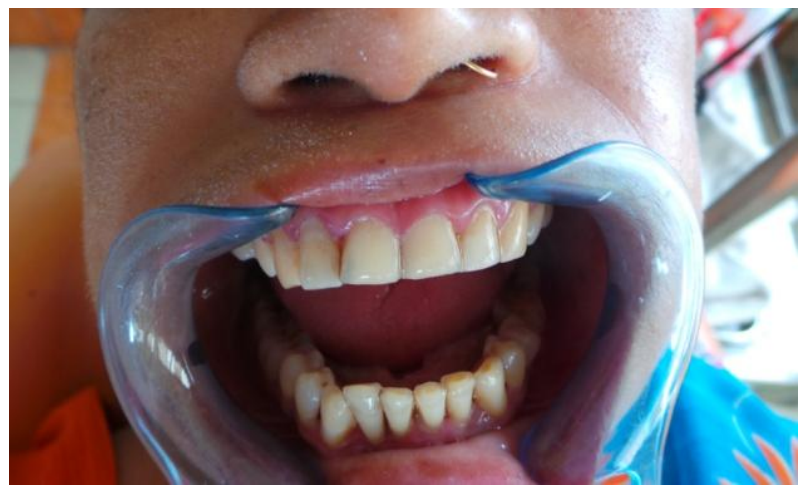

Fig.7: Immediate post operative view 


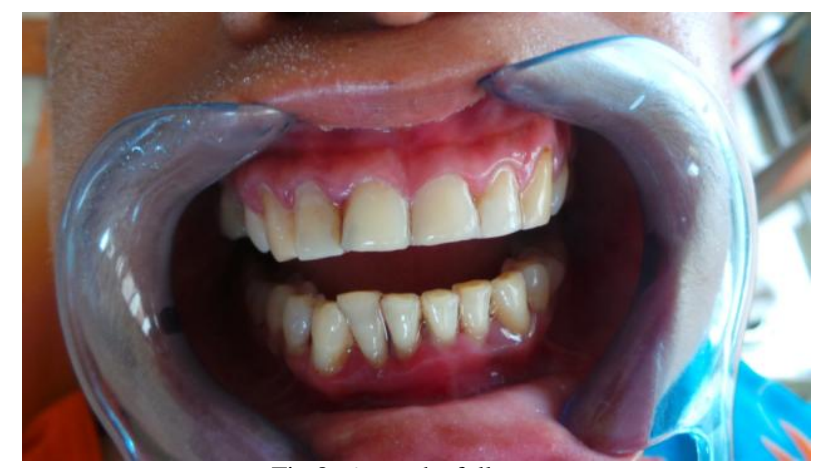

Fig.8: 6 months follow up

\section{Discussion}

Resin-based composite laminate veneers have been extensively investigated and supported by several clinical and laboratory studies.[7,8,9]. However, there are a few long-term follow-up studies with direct resin-based restorations. The case report presented here depict a clinical case involving direct composite resin restorations which resulted in satisfactory outcome for the patient and dental team. In addition, there was no gingival inflammation observed. In this case report, a self etch system was utilized. Although Bekes et al.[10] showed no differences between the total-etch and self-etch in Class I and II restorations, studies have shown that total-etch systems were more successful than self-etch system for enamel bonding.[11,12].

The use of the direct laminate veneer technique with adhesive bonding systems and composite resin materials in this case report is shown with good esthetic result.

\section{Conclusion}

The use of direct resin-based composite laminate veneers and adhesive bonding systems has been shown to provide an esthetic alternative to metal-ceramic or all-ceramic crowns for the rehabilitation of anterior teeth. This treatment option offers another advantage, namely a lower cost compared to an indirect technique. Other more complex and costly treatment options in the future are not ruled out. A significant advantage of

resin-based restorations, over other restorative materials, is that repairs may be possible intraorally without the risk of modifying esthetics or mechanical performance. The unique sculpting instrument gives the ease to manipulate the composite resin with superior handling properties.

\section{Acknowledgements}

I thank IVOCLAR VIVADENT for their instrumental and material support

\section{References}

[1] Deliperi S, Bardwell DN, Congiu MD. Reconstruction of severely damaged endodontically treated and bleached teeth using a microhybrid composite resin: two-year case report. Pract Proced Aesthet Dent. 2003; 15(3):221-6.

[2] Terry DA. Adhesive reattachment of a tooth fragment: the biological restoration. Pract Proced Aesthet Dent. 2003; 15(5):403-9.

[3] Zorba YO, Ozcan E. Reattachment of coronal fragment using fiber-reinforced post: a case report. Eur J Dent. 2007; 1(3):174-8.

[4] Adanir N, Ok E, Erdek Y. Re-attachment of subgingivally oblique fractured central incisor using a fiber post. Eur J Dent. $2008 ; 2$ (2): 138-41.

[5] Bulbule NS, Motwani BK, Tunkiwala A, Pakhan AJ. Esthetic rehabilitation with laminate veneers. J Indian Prosthodont Soc 2006; 6(2):101-4.

[6] Schmidseder J, Aesthetic dentistry. New York: Thieme; 2000. p. 125-31, 206-7.

[7] Izgi AD, Ayna E. Direct restorative treatment of peg-shaped maxillary lateral incisors with resin composite: a clinical report. J Prosthet Dent. 2005; 93(6):526-9.

[8] Belli S, Tanriverdi FF, Belli E. Colour stability of three esthetic laminate materials against to [sic] different staining agents. J Marmara Univ Dent Fac. 1997; 2(4):643-8.

[9] Stappert CF, Ozden U, Gerds T, Strub JR. Longevity and failure load of ceramic veneers with different preparation designs after exposure to masticatory simulation. J Prosthet Dent. 2005; 94(2):132-9. 
[10] Bekes K, Boeckler L, Gernhardt CR, Schaller HG. Clinical performance of a self-etching and a total-etch adhesive system-2-year results. J Oral Rehabil. 2007; 34(11):855-61.

[11] Erickson RL, De Gee AJ, Feilzer AJ. Fatigue testing of enamel bonds with self-etch and total-etch adhesive systems. Dent Mater. 2006; 22(11):981-7.

[12] Strydom C. Self-etching adhesives: review of adhesion to tooth structure part I. SADJ. 2004; 59(10):413, 415-7, 419. 